\title{
A new class of activation functions. Some related problems and applications
}

\author{
Nikolay Kyurkchiev ${ }^{1,2}$ \\ ${ }^{1}$ Faculty of Mathematics and Informatics \\ University of Plovdiv Paisii Hilendarski \\ 24 Tzar Asen Str., 4000 Plovdiv, Bulgaria \\ nkyurk@uni-plovdiv.bg \\ ${ }^{2}$ Institute of Mathematics and Informatics \\ Bulgarian Academy of Sciences \\ Acad. G. Bonchev Str., Bl. 8, 1113 Sofia, Bulgaria
}

Received: 21 January 2020, accepted: 3 May 2020, published: 17 May 2020

\begin{abstract}
The cumulative distribution function (cdf) of the discrete two-parameter bathtub hazard distribution has important role in the fields of population dynamics, reliability analysis and life testing experiments. Also of interest to the specialists is the task of approximating the Heaviside function by new (cdf) in Hausdorff sense. We define new activation function and family of new recurrence generated functions and study the "saturation" by these families. In this paper we analyze some intrinsic properties of the new Topp-Leone-G-Family with baseline "deterministic-type" (cdf) - (NTLGDT). Some numerical examples with real data from Biostatistics, Population dynamics and Signal theory, illustrating our results are given. It is shown that the study of the two characteristics - "confidential curves" and "super saturation" is a must when choosing the right model. Some related problems are discussed, as an example to the Approximation Theory.
\end{abstract}

Keywords-two-parameter bathtub hazard dis- tribution; "saturation" by: new activation function and family of new recurrence generated functions; Topp-Leone-G-Family with baseline "deterministic-type" (cdf) - (NTLG-DT); Heaviside function; Hausdorff distance; upper and lower bounds

\section{INTRODUCTION AND PRELIMINARIES}

Definition 1. Define the following deterministic (cdf) based on two-parameter bathtub hazard distribution [2]:

$$
M_{\beta}(t)=1-q^{e^{t^{\beta}}-1}
$$

where $0<q<1 ; \beta>0, t>0$.

Definition 2. The shifted Heaviside step function

Copyright: (c) 2020 Kyurkchiev. This article is distributed under the terms of the Creative Commons Attribution License (CC BY 4.0), which permits unrestricted use, distribution, and reproduction in any medium, provided the original author and source are credited.

Citation: Nikolay Kyurkchiev, A new class of activation functions. Some related problems and applications, Biomath 9 (2020), 2005033, http://dx.doi.org/10.11145/j.biomath.2020.05.033 
Nikolay Kyurkchiev, A new class of activation functions. Some related problems and applications

is defined by

$$
h_{t_{0}}(t)=\left\{\begin{array}{cll}
0, & \text { if } & t<t_{0}, \\
{[0,1],} & \text { if } & t=t_{0}, \\
1, & \text { if } & t>t_{0}
\end{array}\right.
$$

Definition 3. [3] The Hausdorff distance (the $H_{-}$ distance) $\rho(f, g)$ between two interval functions $f, g$ on $\Omega \subseteq \mathbb{R}$, is the distance between their completed graphs $F(f)$ and $F(g)$ considered as closed subsets of $\Omega \times \mathbb{R}$. More precisely,

$$
\begin{gathered}
\rho(f, g)=\max \left\{\sup _{A \in F(f)} \inf _{B \in F(g)}\|A-B\|,\right. \\
\left.\sup _{B \in F(g)} \inf _{A \in F(f)}\|A-B\|\right\},
\end{gathered}
$$

wherein $\|$.$\| is any norm in \mathbb{R}^{2}$, e. g. the maximum norm $\|(t, x)\|=\max \{|t|,|x|\}$; hence the distance between the points $A=\left(t_{A}, x_{A}\right), B=\left(t_{B}, x_{B}\right)$ in $\mathbb{R}^{2}$ is $\|A-B\|=\max \left(\left|t_{A}-t_{B}\right|,\left|x_{A}-x_{B}\right|\right)$.

Definition 4. We define the following activation function:

$$
A(t ; \beta)=\frac{q^{e^{-t^{\beta}}}-q^{e^{t^{\beta}}}}{q^{e^{-t^{\beta}}}+q^{e^{t^{\beta}}}} .
$$

Definition 5. Define the following family of new recurrence generated functions

$$
\begin{aligned}
& A_{i+1}(t ; \beta)=A_{i}\left(t+A_{i}(t ; \beta) ; \beta\right), \\
& i=0,1,2, \ldots ; A_{0}(t ; \beta)=A(t ; \beta) .
\end{aligned}
$$

based on the function $A(t ; \beta)$.

In [1] Bantan, Jamal, Chesneau and Elgarhy introduced a new power Topp-Leone-G-Family (NTL-G) of distribution with (cdf)

$$
F(t)=e^{\alpha \beta\left(1-\frac{1}{G(t)}\right)}\left(2-e^{\beta\left(1-\frac{1}{G(t)}\right)}\right)^{\alpha}
$$

where $\alpha, \beta \in R^{+}$and $G(t)$ is a (cdf) of a baseline continuous distribution.
The following result shows some inequalities involving $F(t)$ (see, Proposition 1 [1]):

$e^{\alpha \beta\left(1-\frac{1}{G(t)}\right)}\left(2-G(t)^{\beta}\right)^{\alpha} \leq F(t) \leq 2^{\alpha} e^{\alpha \beta\left(1-\frac{1}{G(t)}\right)}$

In this paper we study some properties of the new Topp-Leone-G-Family with baseline "deterministic-type" (cdf) - (NTLG-DT); $G(t)=$ $1-q^{e^{t}-1}$, where $0<q<1$.

Definition 6. We define the following corresponding $(c d f)$ :

$$
Q(t)=e^{\alpha \beta\left(1-\frac{1}{1-q^{e^{t}}-1}\right)}\left(2-e^{\beta\left(1-\frac{1}{1-q^{e^{t}-1}}\right)}\right)^{\alpha}
$$

where $\alpha, \beta \in R^{+}$and $0<q<1$.

\section{MAin Results}

When studying the intrinsic properties of the family $M_{\beta}(t)$, it is also appropriate to study the "saturation" to the horizontal asymptote.

In this Section we give upper and lower estimates for the one-sided Hausdorff approximation of the Heaviside step-function $h_{t_{0}}(t)$ by means of family (1), where $t_{0}$ is the level of the "median".

A. The case $\beta=1$.

Let $t_{0}$ is the unique positive root of the nonlinear equation $M_{1}\left(t_{0}\right)-\frac{1}{2}=0$.

The one-sided Hausdorff distance $d$ between $h_{t_{0}}(t)$ and the function (1) satisfies the relation

$$
M_{1}\left(t_{0}+d\right)=1-q^{e^{\left(t_{0}+d\right)}-1}=1-d .
$$

The following theorem gives upper and lower bounds for $d$

Theorem 1. Let

$$
\begin{aligned}
& \beta=1, \\
& q<\frac{2}{e^{2\left(\frac{e^{1.05}}{2.1}-1\right)}} \approx 0.971975 .
\end{aligned}
$$

Then, for the one-sided Hausdorff distance $d$ between $h_{t_{0}}(t)$ and the (cdf) - (1) the following inequalities hold:

$$
d_{l}=\frac{1}{2.1\left(1+\frac{1}{2} \ln \frac{2}{q}\right)}<d<\frac{\ln \left(2.1\left(1+\frac{1}{2} \ln \frac{2}{q}\right)\right)}{2.1\left(1+\frac{1}{2} \ln \frac{2}{q}\right)}=d_{r} .
$$


Nikolay Kyurkchiev, A new class of activation functions. Some related problems and applications

Proof. In order to express $d$ in terms of $q$, let us examine the function

$$
f(d)=M_{1}\left(t_{0}+d\right)-1+d .
$$

From $f^{\prime}(d)>0$ we conclude that function $f(d)$ is strictly monotonically increasing.

Consider then the function

$$
g(d)=-\frac{1}{2}+\left(1+\frac{1}{2} \ln \frac{2}{q}\right) d,
$$

which approximates function $f$ with $d \rightarrow 0$ as $O\left(d^{2}\right)$ (see, Fig. 1).

In addition $g^{\prime}(d)>0$.

We look for two reals $d_{l}$ and $d_{r}$ such that $g\left(d_{l}\right)<0$ and $g\left(d_{r}\right)>0$ (leading to $g\left(d_{l}\right)<$ $\left.d<g\left(d_{r}\right)\right)$.

From (9) we have

$$
\begin{gathered}
g\left(d_{l}=\frac{1}{2.1\left(1+\frac{1}{2} \ln \frac{2}{q}\right)}\right)<0, \\
g\left(d_{r}=\frac{\ln \left(2.1\left(1+\frac{1}{2} \ln \frac{2}{q}\right)\right)}{2.1\left(1+\frac{1}{2} \ln \frac{2}{q}\right)}\right)>0
\end{gathered}
$$

proving the estimates (10).

For example, for $\beta=1, q=0.1$ we have

$$
d_{l}=0.190639<d=0.230226<0.31596=d_{r}
$$

and for $\beta=1, q=0.9$ we have

$$
d_{l}=0.340317<d=0.355551<0.36682=d_{r} .
$$

\section{B. The case $\beta \neq 1$.}

For given $\beta \neq 1$ the one-sided Hausdorff distance $d$ satisfies the relation

$$
M_{\beta}\left(t_{0}+d\right)=1-q^{e^{\left(t_{0}+d\right)^{\beta}}-1}=1-d .
$$

The reader may formulate the corresponding approximation problem following the ideas given in Theorem 1, and will be omitted.

We illustrate the "saturation" with the (cdf) (1) for various $\beta$ and fixed $q=0.1$ (see, Fig. 2)

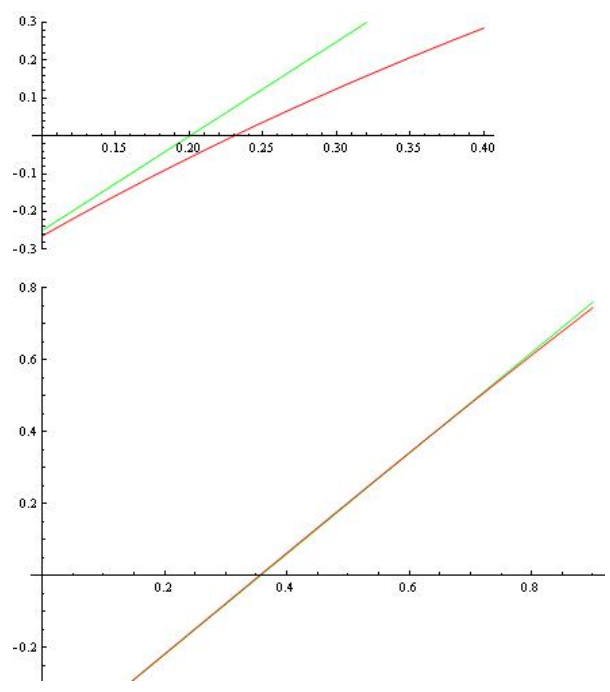

Fig. 1. The functions $f(d)$ and $g(d)$ for a) $\beta=1, q=0.1$; b) $\beta=1, q=0.9$.
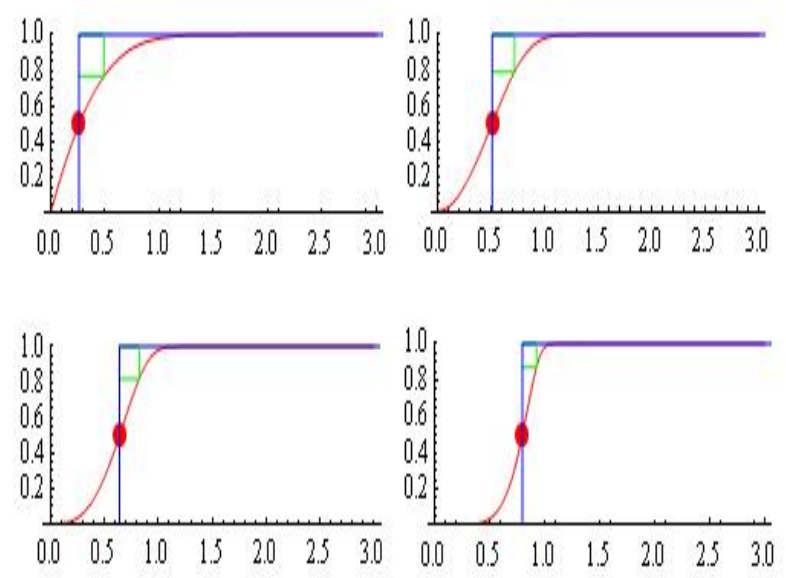

Fig. 2. a) $\beta=1, q=0.1 ; t_{0}=0.263156$; Hausdorff distance $d=0.230226$; b) $\beta=2, q=0.1 ; t_{0}=0.512988$; Hausdorff distance $d=0.208046$; c) $\beta=3, q=0.1 ; t_{0}=$ 0.640823 ; Hausdorff distance $d=0.181048$; d) $\beta=6, q=$ $0.1 ; t_{0}=0.800514$; Hausdorff distance $d=0.127635$.

\section{Some ApplicAtions.}

It is well known that in many cases the existing modifications to the classical logistic and Gompertz models do not give very reliable results in approximating "specific data".

We examine the following "specific datasets": 


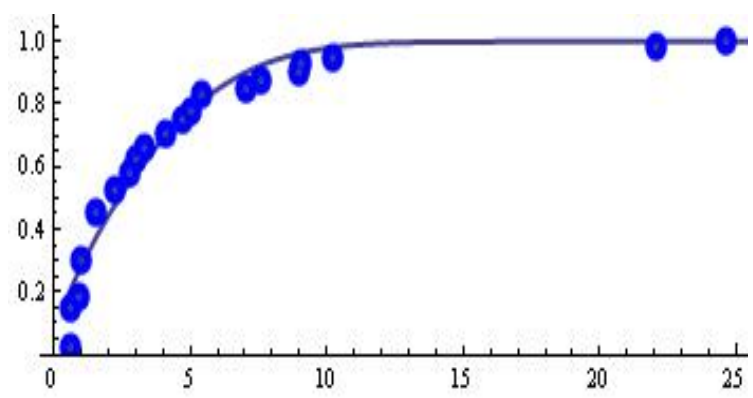

Fig. 3. The fitted model (1).

Example 1. We analyze the following data [4] data_Communication $:=\{\{0.584,0.027\}$, $\{0.649,0.147\},\{0.909,0.187\},\{1.039,0.303\}$, $\{1.558,0.453\},\{2.208,0.527\},\{2.792,0.580\}$, $\{3.052,0.627\},\{3.312,0.657\},\{4.091,0.707\}$, $\{4.740,0.753\},\{5,0.780\},\{5.390,0.827\}$, $\{7.078,0.853\},\{7.597,0.877\},\{8.961,0.903\}$, $\{9.091,0.927\},\{10.195,0.950\},\{22.078,0.980\}$, $\{24.610,1\}\}$;

The cdf $M_{\beta}(t)$ for $\beta=0.484411$ and $q=$ 0.82547 is visualized on Fig. 3 .

Example 2. Analysis of "data_Nicotine" [5] data_Nicotine $:=$

$$
\begin{aligned}
& \{\{0.11,0.021\},\{0.21,0.053\},\{0.31,0.063\}, \\
& \{0.41,0.105\},\{0.51,0.2\},\{0.61,0.274\}, \\
& \{0.71,0.358\},\{0.81,0.495\},\{0.91,0.632\}, \\
& \{1.01,0.726\},\{1.11,0.832\},\{1.21,0.905\}, \\
& \{1.31,0.942\},\{1.41,0.958\},\{1.51,0.974\}, \\
& \{1.61,0.979\},\{1.71,0.989\},\{1.81,1\}, \\
& \{1.9,1\},\{2,1\}\}
\end{aligned}
$$

After that using the model $M_{\beta}(t)$ for $\beta=1.98567$ and $q=0.485475$ we obtain the fitted model (see, Fig. 4).

Example 3. Analysis of data "Biomass produced by Paesilomyces lilacinus 6029" [6].

After that using the model $M_{\beta}^{*}(t)=\omega M_{\beta}(t)$ for $\omega=10.521, \beta=0.805824$ and $q=0.97915$ we obtain the fitted model (see, Fig. 5).

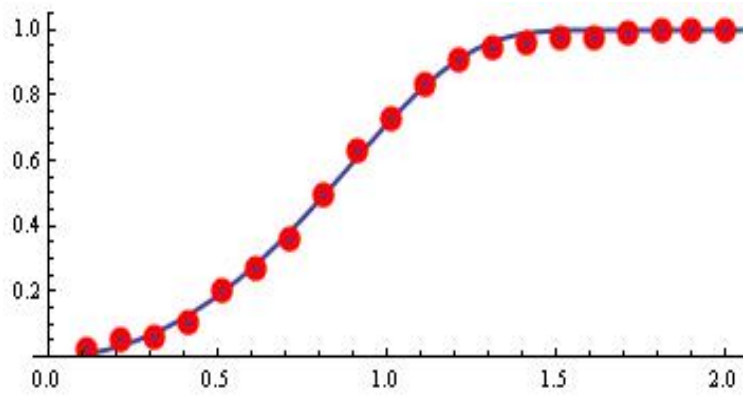

Fig. 4. The fitted model (1).

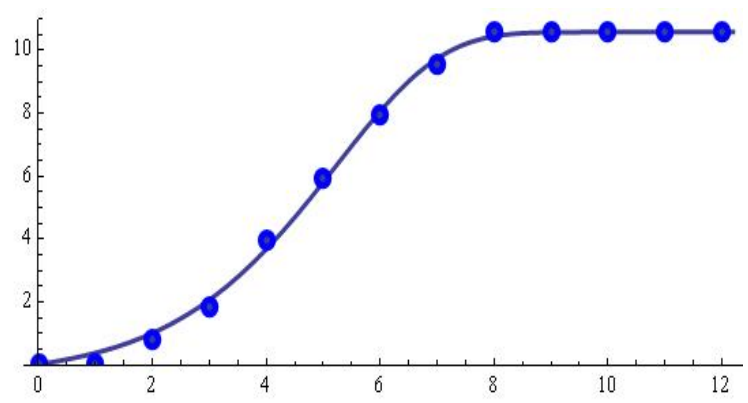

Fig. 5. The fitted model.

The new activation function.

We define the following activation function:

$$
A(t ; \beta)=\frac{q^{e^{-t^{\beta}}}-q^{e^{t^{\beta}}}}{q^{e^{-t^{\beta}}}+q^{e^{t^{\beta}}} .}
$$

In antenna-feeder technique most often occurred signals are of types shown on Fig. 6 - Fig. 7.

For $\beta$ even, the corresponding approximation using model (7) is shown in Fig. 6.

For $\beta$ odd, the corresponding approximation using new activation function $A(t ; \beta)$ is shown in Fig. 7.

A family of recurrence generated functions based on the $A(t ; \beta)$.

Let us consider the following family of recurrence generated functions

$$
\begin{aligned}
& A_{i+1}(t ; \beta)=A_{i}\left(t+A_{i}(t ; \beta) ; \beta\right), \\
& i=0,1,2, \ldots ; A_{0}(t ; \beta)=A(t ; \beta),
\end{aligned}
$$

based on the function $A(t ; \beta)$.

Let for instance $\beta=1$. 

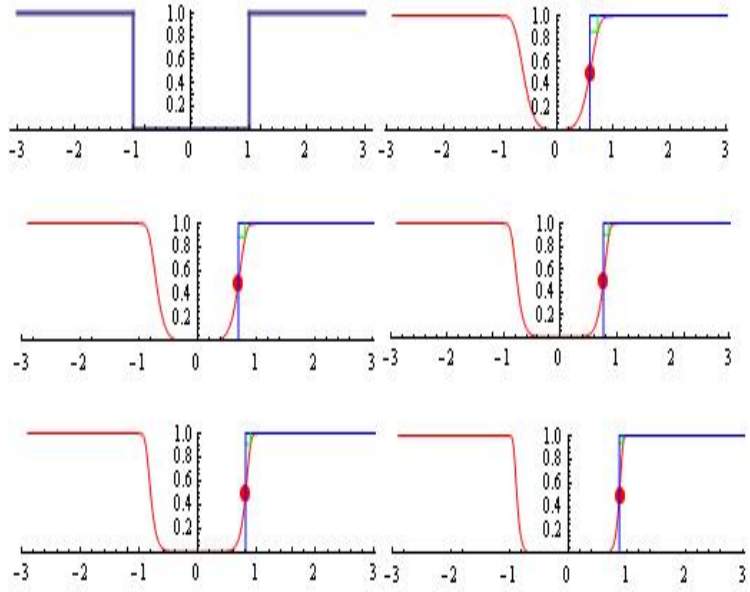

Fig. 6. The function $A(t ; \beta) ; \beta=4, q=0.01, t_{0}=$ 0.587335 ; Hausdorff distance $d=0.138899 ; \beta=6, q=$ $0.01, t_{0}=0.701333 ;$ Hausdorff distance $d=0.111603$; $\beta=8, q=0.01, t_{0}=0.766378$; Hausdorff distance $d=$ $0.0992629 ; \beta=10, q=0.01, t_{0}=0.808266$; Hausdorff distance $d=0.0867535 ; \beta=16, q=0.01, t_{0}=0.87543$; Hausdorff distance $d=0.0632673$.
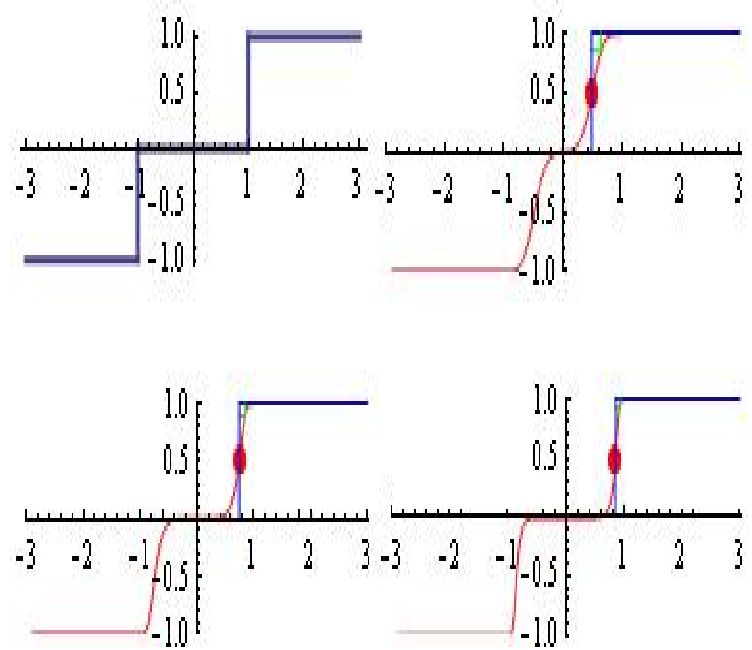

Fig. 7. The function $A(t ; \beta) ; \beta=3, q=0.01, t_{0}=$ 0.491867; Hausdorff distance $d=0.152538 ; \beta=7, q=$ $0.01, t_{0}=0.737794$; Hausdorff distance $d=0.107003$; $\beta=13, q=0.01, t_{0}=0.848962$; Hausdorff distance $d=0.073086$.
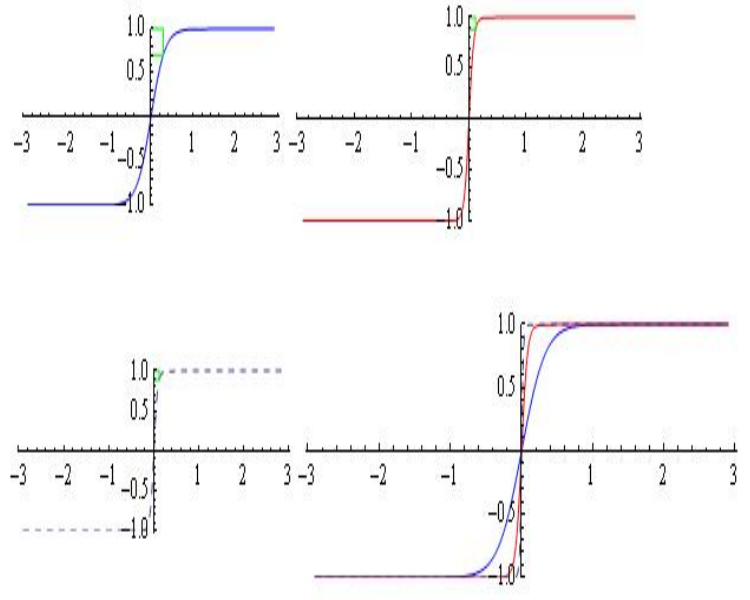

Fig. 8. The recurrence generated family: $A_{0}(t)$ (blue), $A_{1}(t)$ (red) and $A_{2}(t)$ (dashed).

The recurrence generated family: $A_{0}(t), A_{1}(t)$ and $A_{2}(t)$ is visualized on Fig. 8.

Some properties of the new Topp-Leone-GFamily with baseline "deterministic-type" (cdf) (NTLG-DT) $Q(t)(7)$.

We study the Hausdorff approximation of the Heaviside step function $h_{t_{0}}(t)$ where $t_{0}$ is the "median" by families of the new Topp-Leone-GFamily with baseline "deterministic-type" (cdf) (NTLG-DT).

The obtained two-sides estimations (see Proposition 1. [1] ) in particular case with usage of the baseline "deterministic-type" (cdf) for $\alpha=$ $0.9 ; \beta=0.3 ; q=0.1$

$$
\begin{aligned}
& e^{\alpha \beta\left(1-\frac{1}{1-q^{e^{t}}-1}\right)}\left(2-\left(1-q^{e^{t}-1}\right)^{\beta}\right)^{\alpha} \leq Q(t) \\
& \leq 2^{\alpha} e^{\alpha \beta\left(1-\frac{1}{1-q^{e^{t}}-1}\right)}
\end{aligned}
$$

are given in Fig. 9 a.

Let $t_{0}$ is the value for which $Q\left(t_{0}\right)=\frac{1}{2}$.

The Hausdorff distance $d$ between the function $h_{t_{0}}(t)$ and $Q(t)$ satisfies the relation

$$
Q\left(t_{0}+d\right)=1-d
$$



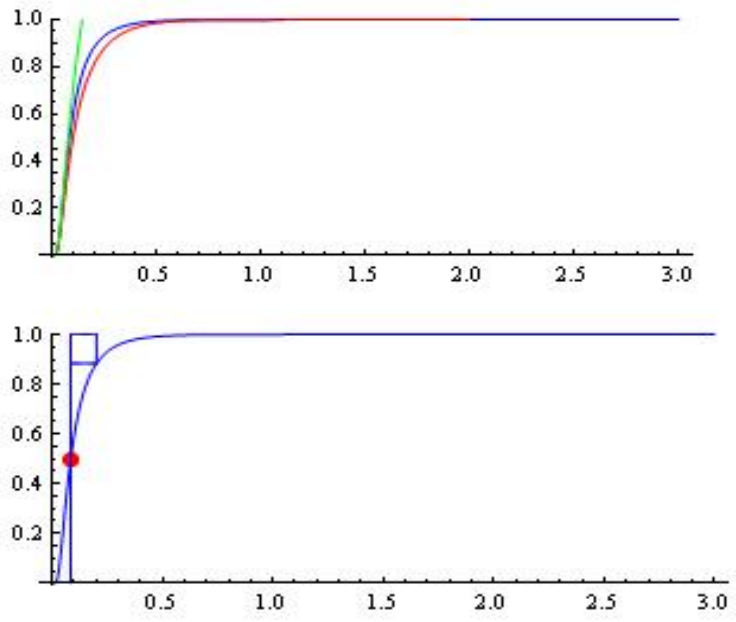

Fig. 9. a) The two-sides estimations (14) for $\alpha=0.9 ; \beta=$ $0.3 ; q=0.1$; b) The model $Q(t)$ for $\alpha=0.9 ; \beta=0.3 ; q=$ $0.1, t_{0}=0.0852097 ;$ H-distance $d=0.116811$

For fixed $\alpha=0.9 ; \beta=0.3 ; q=0.1$ we find $t_{0}=0.0852097$ and from the nonlinear equation (15) we have $d=0.116811$ (see, Fig. 9 b).

From Fig. 9 it can be seen that these estimations can be used as "confidence bounds", which are extremely useful for the specialists in the choice of model for cumulative data approximating in areas of Biostatistics, Population dynamics, Growth theory, Debugging and Test theory, Computer viruses propagation, Financial and Insurance mathematics.

For other results, see [8]-[53], [59].

\section{CONCLUDING REMARKS.}

The results obtained in this article can be successfully continued.

1. For example, we study the Hausdorff approximation of the Heaviside step function $h_{t_{0}}(t)$ where $t_{0}$ is the "median" by families of the new Topp-Leone-G-Family $Q_{1}(t)$ with baseline "deterministic-inverse-type" (cdf) - (NTLG-DIT) $G(t)=q^{e^{\frac{1}{t}}-1}$, where $0<q<1$,

$$
Q_{1}(t)=e^{\alpha \beta\left(1-\frac{1}{q^{e^{\frac{1}{t}}}-1}\right)}\left(2-e^{\beta\left(1-\frac{1}{q^{e^{\frac{1}{t}}}-1}\right)}\right)^{\alpha}
$$
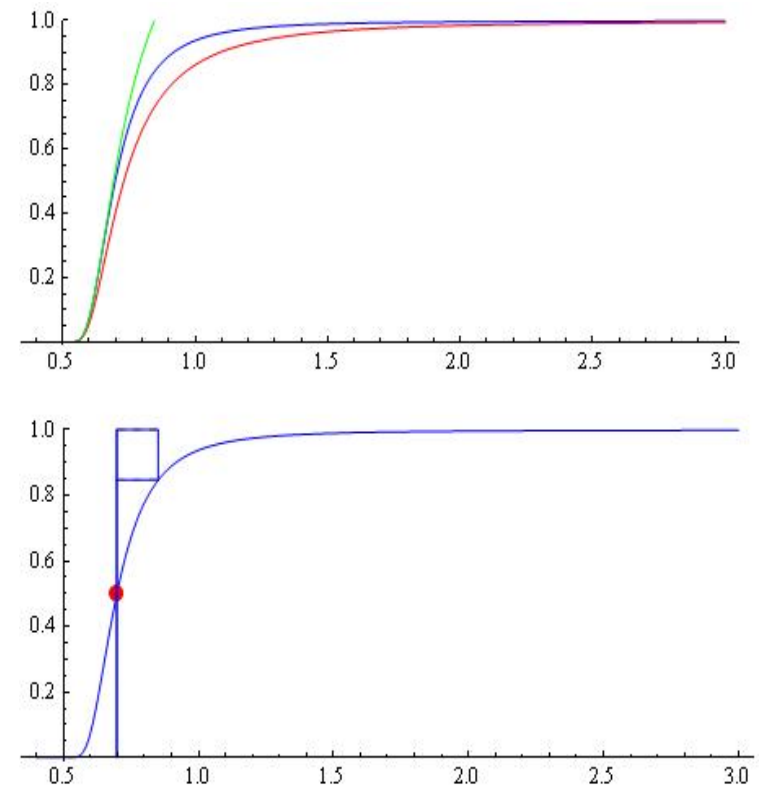

Fig. 10. a) The two-sided bounds (17) for $\alpha=0.6 ; \beta=$ $0.1 ; q=0.4$; b) The model $Q_{1}(t)$ for $\alpha=0.6 ; \beta=0.1 ; q=$ $0.4, t_{0}=0.698075 ; \mathrm{H}$-distance $d=0.153113$

The obtained two-sided bounds (see Proposition 1. [1] ) in particular case with usage of the baseline "deterministic-inverse-type" (cdf) for $\alpha=$ $0.6, \beta=0.1, q=0.4$,

$$
\begin{gathered}
e^{\alpha \beta\left(1-\frac{1}{q^{e^{\frac{1}{t}}}-1}\right)}\left(2-q^{\beta\left(e^{\frac{1}{t}}-1\right)}\right)^{\alpha} \\
\leq Q_{1}(t) \leq 2^{\alpha} e^{\alpha \beta\left(1-\frac{1}{q^{e^{\frac{1}{t}}}-1}\right)}
\end{gathered}
$$

are given in Fig. 10 a.

Example 4. Storm worm one of the most biggest cyber threats of 2008 .

We analyze the following data [7]

$$
\begin{aligned}
& \text { data_Storm_IDs }:=\{\{1,0.843\}, \\
& \{4,0.926\},\{5,0.954\},\{6,0.967\}, \\
& \{7,0.976\},\{8,0.981\},\{9,0.985\}, \\
& \{10,0.991\},\{22,0.995\},\{38,0.997\}, \\
& \{51,0.998\},\{64,0.9985\},\{74,0.999\}, \\
& \{83,1\},\{100,1\},\{367,1\}\}
\end{aligned}
$$




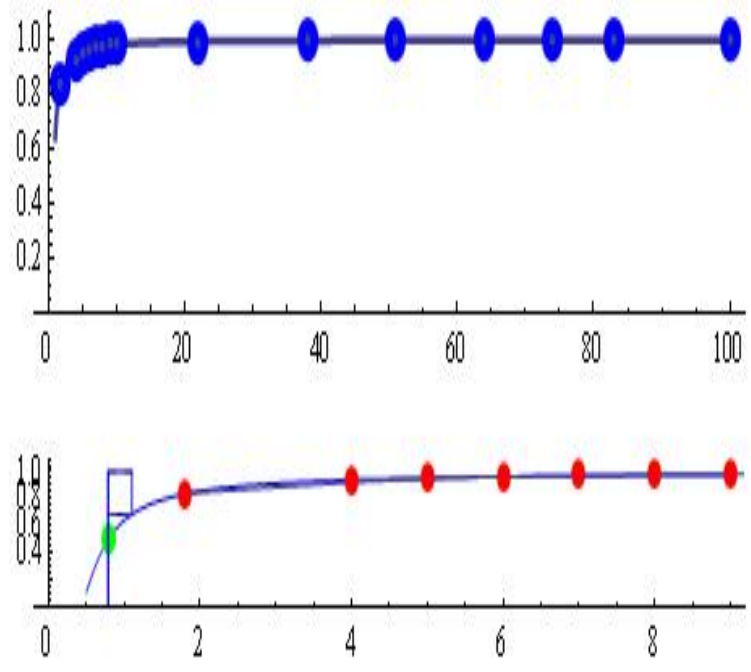

Fig. 11. The fitted model $Q_{1}(t)$.

The cdf $Q_{1}(t)$ for $\alpha=0.14146, \beta=$ 161.078891, $q=0.9$ is visualized on Fig. 11.

Exploring both features - "confidential curves" and "super saturation" is a must when choosing the right model.

2. Following the ideas given in $[54]-[56]$ we consider the following new differential model:

$$
\left\{\begin{array}{l}
\frac{d y(t)}{d t}=k y(t) s(t)=k y(t) q^{e^{t}-1} \\
y\left(t_{0}\right)=y_{0}
\end{array}\right.
$$

where $k>0$ and $0<q<1$.

The general solution of the differential equation (18) is of the following form:

$$
y(t)=y_{0} e^{\frac{k}{q} E i\left(e^{t} \ln q\right)-\frac{k}{q} E i(\ln q)}
$$

where $E i($.$) is the traditional exponential integral.$

The new "growth" function $y(t)$ and the "input function" $s(t)=q^{e^{t}-1}$ are visualized on Fig. 12Fig. 13.

Example 4. We will analyze a sample of experimental data obtained by the biologist T. Carlson in 1913 about the development of Saccharomyces culture in nutrient medium (see, for example [58], [57]).

After that using the model $M^{*}(t)=$ $\omega e^{\frac{k}{q} E i\left(e^{t} \ln q\right)-\frac{k}{q} E i(\ln q)}$ for $k=0.293574$,

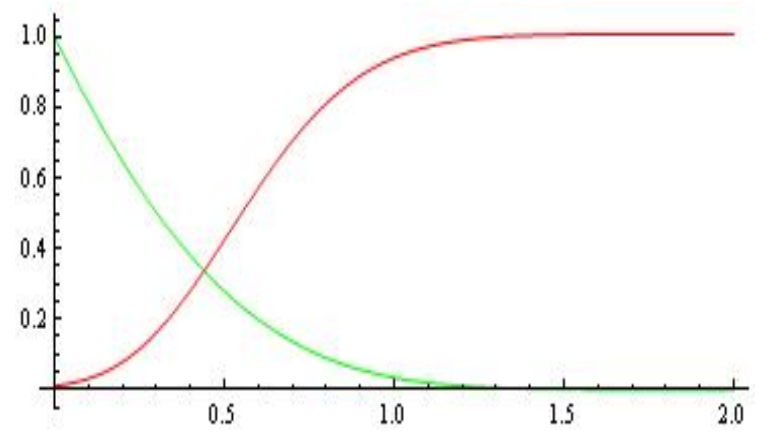

Fig. 12. The "growth" function $y(t)-($ red) and $s(t)-($ green) for $k=12.6 ; q=0.14 ; y_{0}=0.01$.

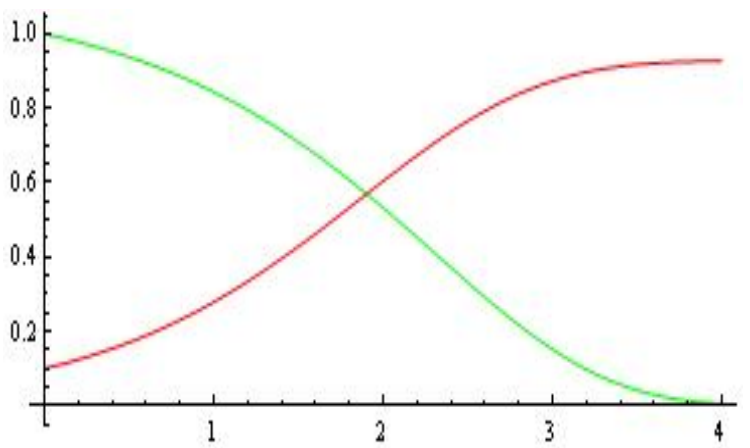

Fig. 13. The "growth" function $y(t)-($ red) and $s(t)-($ green) for $k=1.1 ; q=0.906 ; y_{0}=0.1$.

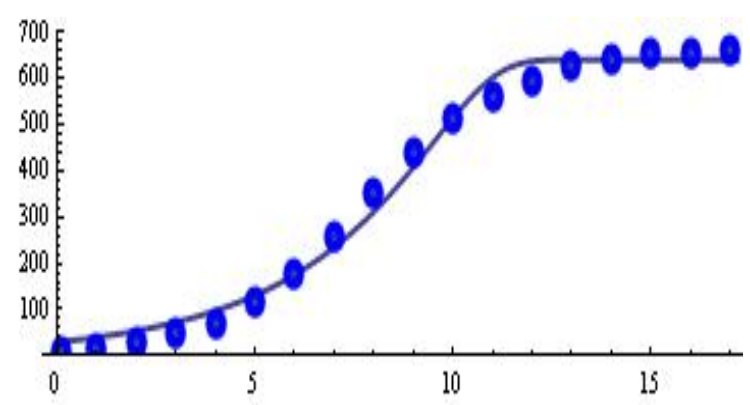

Fig. 14. The fitted model $M^{*}(t)$. 


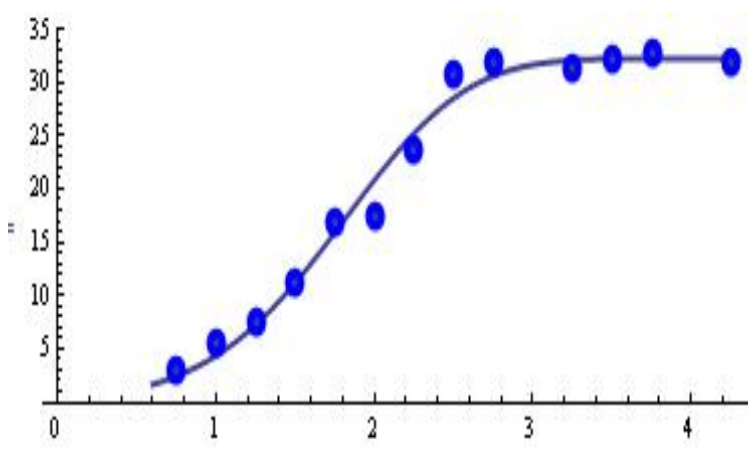

Fig. 15. The fitted model $M^{*}(t)$.

$q=0.999983$ and $\omega=30.114$ we obtain the fitted model (see, Fig. 14).

Example 5. Analysis of data "Biomass produced by Paesilomyces sinclairi ascomycota".

After that using the model $M^{*}(t)$ for $\omega=$ $0.305247, k=3.01914$ and $q=0.83$ we obtain the fitted model (see, Fig. 15).

The general solution $y(t)$ has been applied widely in life testing experiments and debugging theory.

\section{ACKNOWLEDGMENT}

The author would like to thank the anonymous referees for their valuable comments.

This paper is supported by the National Scientific Program "Information and Communication Technologies for a Single Digital Market in Science, Education and Security (ICTinSES)", financed by the Ministry of Education and Science.

\section{REFERENCES}

[1] R. Bantan, F. Jamal, Ch. Chesneau, M. Elgarhy, A New Power Topp-Leone Generated Family of Distributions with Applications, Entropy, 21, 12, 1177, 2019.

[2] A. Sarhan, Bayesian analysis of the discrete twoparameter bathtub hazard distribution, J. of Math. Sci. and Modelling, 2, 3, 183-192, 2019.

[3] B. Sendov, Hausdorff Approximations, Kluwer, Boston, 1990.

[4] P. E. Oguntunde, M. A. Khaleel, H. I. Okagbue, O. A. Odetunmibi, The Topp-Leone Lomax (TLLo) Distribution with Applications to Airbone Communication Transceiver Dataset, Wireless Personal Communications, 109, 349360, 2019.
[5] A. Afify, A. Abdellatif, The extended Burr XII distribution: properties and applications, J. of Nonlinear Sci. and Appl., 13, 133-146, 2020.

[6] A. Sharma, S. Sharma, A. Mittal, S. Naik, Statistical optimization of growth media for Paecilomyces lilacinus 6029 using non-edible oil cakes, Ann. of Microbiology, 64, 515-520, 2014.

[7] S. Sarat, A. Terzis, HiNRG Technical Report: 01-10-2007 Measuring the Storm Worm Network, 2007.

[8] B. Gompertz, On the nature of the function expressive of the law of human mortality, and on a new mode of determining the 62 value of life contingencies, Philos Trans R Soc London, 115, 513-585, 1825.

[9] S. Markov, Reaction networks reveal new links between Gompertz and Verhulst growth functions, Biomath, 8, 1, 2019.

[10] L. Rogers-Bennett, D. W. Rogers, A Two-Step Growth Curve: Approach to the von Bertalanffy and Gompertz Equations, Advances in Pure Mathematics, 6, 321-330, 2016.

[11] K. Tjrve, E. Tjrve, The use of Gompertz models in growth analyses, and new Gompertz-model approach: An addition to the Unified-Richards family, PLoS ONE, 12, 6, 2017.

[12] M. Tahir, G. Cordeiro, Compounding of distributions: A survey and new generalized classes, J. of Stat. Distr. and Appl., 3, 13, 1-35, 2016.

[13] D. Satoh, Property of logistic data exposed with Gompertz model and resistance to noise in actual data, Japan J. of Ind. and Appl. Math., 2019.

[14] S. Yamada, A stochastic software reliability growth model with Gompertz curve, Trans. IPSJ, 33, 964-969, 1992. (in Japanese)

[15] D. Satoh, S. Yamada, Discrete equations and software reliability growth models, in: Proc. 12th Int. Symp. on Software Reliab. and Eng., 176-184, 2001.

[16] H. Okamura, T. Dohi, S. Osaki, EM algorithms for logistic software reliability models, In: Proc. 7th IASTED Int. Conf. on Software Eng., 263-268, 2004.

[17] S. Yamada, M. Ohba, S. Osaki, S-shaped reliability growth modeling for software error detection, IEEE Trans, Reliab., R-32, 475-478, 1983.

[18] M. Xie, Software Reliability Modelling, World Scientific, Singapore, 1991.

[19] H. Pham, Software Reliability, Springer, Singapore, 2000.

[20] N. Kyurkchiev, On a sigmoidal growth function generated by reaction networks. Some extensions and applications, Communications in Applied Analysis, 23, 3, 383-400, 2019.

[21] D. Costarelli, R. Spigler, Constructive Approximation by Superposition of Sigmoidal Functions, Anal. Theory Appl., 29, 169-196, 2013.

[22] D. Costarelli, G. Vinti, Pointwise and uniform approximation by multivariate neural network operators of the max-product type, Neural Networks, 81, 81-90, 2016.

[23] N. Guliyev, V. Ismailov, A single hidden layer feed- 
forward network with only one neuron in the hidden layer san approximate any univariate function, Neural Computation, 28, 1289-1304, 2016.

[24] N. Kyurkchiev, S. Markov, On the Hausdorff distance between the Heaviside step function and Verhulst logistic function, J. Math. Chem., 54, 1, 109-119, 2016.

[25] N. Kyurkchiev, S. Markov, Sigmoid functions: Some Approximation and Modelling Aspects, LAP LAMBERT Academic Publishing, Saarbrucken, 2015, ISBN 978-3659-76045-7.

[26] N. Kyurkchiev, A. Iliev, S. Markov, Some Techniques for Recurrence Generating of Activation Functions: Some Modeling and Approximation Aspects, LAP LAMBERT Academic Publishing, 2017, ISBN: 978-3-330-33143-3.

[27] A. Iliev, N. Kyurkchiev, S. Markov, A Note on the New Activation Function of Gompertz Type, Biomath Communications, 4, 2, 20 pp., 2017.

[28] R. Anguelov, M. Borisov, A. Iliev, N. Kyurkchiev, S. Markov, On the chemical meaning of some growth models possessing Gompertzian-type property, Math. Meth. Appl. Sci., 1-12, 2017.

[29] R. Anguelov, N. Kyurkchiev, S. Markov, Some properties of the Blumberg's hyper-log-logistic curve, Biomath, 7, 1, 8 pp., 2018.

[30] S. Markov, A. Iliev, A. Rahnev, N. Kyurkchiev, On the exponential-generalized extended Compertz cumulative sigmoid, International Journal of Pure and Applied Mathematics, 120, 4, 555-562, 2018.

[31] N. Pavlov, A. Iliev, A. Rahnev, N. Kyurkchiev, Some software reliability models: Approximation and modeling aspects, LAP LAMBERT Academic Publishing, 2018, ISBN: 978-613-9-82805-0.

[32] A. Iliev, N. Kyurkchiev, A. Rahnev, T. Terzieva, Some models in the theory of computer viruses propagation, LAP LAMBERT Academic Publishing, 2019, ISBN: 978-620-0-00826-8.

[33] A. Iliev, N. Kyurkchiev, S. Markov, On the approximation of the cut and step functions by logistic and Gompertz functions, Biomath, 4, 2-13, 2015.

[34] N. Kyurkchiev, A. Iliev, A. Rahnev, A new class of activation functions based on the correcting amendments of Gompertz-Makeham type, Dynamic Systems and Applications, 28, 2, 243-257, 2019.

[35] N. Kyurkchiev, A. Iliev, A. Rahnev, Some Families of Sigmoid Functions: Applications to Growth Theory, LAP LAMBERT Academic Publishing, 2019, ISBN: 978-6139-45608-6.

[36] N. Pavlov, A. Iliev, A. Rahnev, N. Kyurkchiev, Nontrivial Models in Debugging Theory (Part 2), LAP LAMBERT Academic Publishing, 2018, ISBN: 978-613-987794-2.

[37] O. Rahneva, T. Terzieva, A. Golev, Investigations on the Zubair-family with baseline Ghosh-Bourguignon's extended Burr XII cumulative sigmoid. Some applications, Neural, Parallel, and Scientific Computations, 27, 1, 1122, 2019.

[38] A. Malinova, O. Rahneva, A. Golev, V. Kyurkchiev,
Investigations on the Odd-Burr-III-Weibull cumulative sigmoid. Some applications, Neural, Parallel, and Scientific Computations, 27, 1, 35-44, 2019.

[39] N. Kyurkchiev, Investigations on a hyper-logistic model. Some applications, Dynamic Systems and Applications, 28, 2, 351-369, 2019.

[40] S. Markov, A. Iliev, A. Rahnev, N. Kyurkchiev, A note on the n-stage growth model. Overview, Biomath Communications, 5, 2, 79-100, 2018.

[41] E. Angelova, A. Golev, T. Terzieva, O. Rahneva, A study on a hyper-power-logistic model. Some applications, Neural, Parallel, and Scientific Computations, 27, 1, 45-57, 2019.

[42] N. Kyurkchiev, A. Iliev, A. Rahnev, On a special choice of nutrient supply for cell growth in a continuous bioreactor. Some modeling and approximation aspects, Dynamic Systems and Applications, 28, 3, 587-606, 2019.

[43] N. Kyurkchiev, A. Iliev, Extension of Gompertz-type equation in modern science. 240 Anniversary of the birth of B. Gompertz, LAP LAMBERT Academic Publishing, 2018, ISBN: 978-613-9-90569-0.

[44] N. Kyurkchiev, A. Iliev, A. Rahnev, T. Terzieva, Properties of a power Topp-Leone G-family with baseline Gompertz cumulative distribution function, 2020. (in print)

[45] B. I. Yun, A Neural Network Approximation Based on a Parametric Sigmoidal Function, Mathematics, 7, 262, 2019.

[46] N. Kyurkchiev, Comments on the Yun's algebraic activation function. Some extensions in the trigonometric case, Dynamic Systems and Applications, 28, 3, 533543, 2019.

[47] N. Kyurkchiev, A. Andreev, Approximation and Antenna and Filters synthesis. Some Moduli in Programming Environment MATHEMATICA, LAP LAMBERT Academic Publishing, Saarbrucken, 2014, ISBN 978-3-659-533228.

[48] B1. Sendov, H. Schinev, N. Kjurkchiev, Hausdorffsynthesis of aerial grids in scanning the directive diagram, Electropromishlenost i Priboroostroene, 16, 5, 203-205, 1981. (in Bulgarian)

[49] N. Kyurkchiev, Synthesis of slot aerial grids with Hausdorff type directive patterns, $\mathrm{PhD}$ Thesis, Department of Radio-Electronics, VMEI, Sofia, 1979. (in Bulgarian)

[50] N. Kyurkchiev, B1. Sendov, Approximation of a class of functions by algebraic polynomials with respect to Hausdorff distance, Ann. Univ. Sofia, Fac. Math., 67, 573-579, 1975. (in Bulgarian)

[51] N. Kyurkchiev, S. Markov, On the numerical approximation of the "cross" set, Ann. Univ. Sofia, Fac. Math., 66, 19-25, 1974. (in Bulgarian)

[52] J. Peters, Foundations of Computer Vision: Computational Geometry, Visual Image Structures and Object Shape Detection, Springer, Cham, 2017.

[53] N. Kyurkchiev, G. Nikolov, Comments on some new classes of sigmoidal and activation functions. Applications, Dynamic Systems and Applications, 28, 4, 789808, 2019. 
[54] N. Kyurkchiev, S. Markov, On a logistic differential model. Some applications, Biomath Communications, 6, 1, 34-50, 2019.

[55] N. Kyurkchiev, A. Iliev, A. Rahnev, A special choice of nutrient supply for cell growth in logistic differential model. Some applications, Conference Proceedings of AIP, 2019.

[56] N. Kyurkchiev, A. Iliev, A. Golev, A. Rahnev, On a Special Choice of Nutrient Supply with Marshall-Olkin Correction. Some Applications, Communications in Applied Analysis, 23, 3, 401-419, 2019.
[57] B1. Sendov, R. Maleev, S. Markov, S. Tashev, Mathematics for Biologists, University Publishing House "St. Kliment Ohridski”, Sofia, 1991.

[58] E. Bohl, Mathematik in der Biologie, 4., vollständig überarbeitete und erweiterte Auflage, Springer, Berlin, 2006.

[59] N. Kyurkchiev, Selected Topics in Mathematical Modeling: Some New Trends (Dedicated to Academician Blagovest Sendov (1932-2020)), LAP LAMBERT Academic Publishing, 2020; ISBN: 978-620-2-51403-3. 